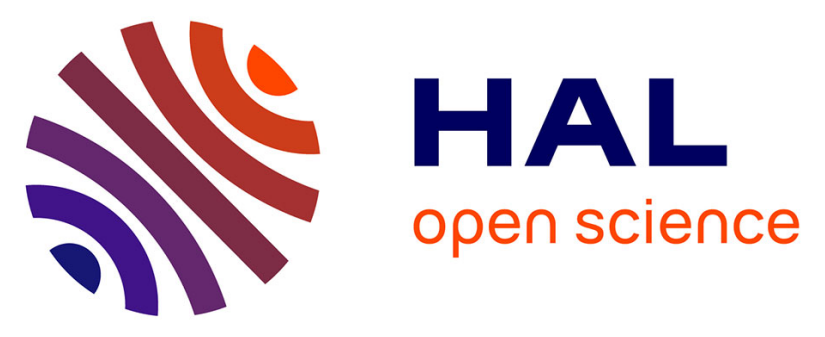

\title{
Experimental Assessment of the Prediction Performance of Dynamic Equivalent Circuit Models of Grid-connected Battery Energy Storage Systems
}

\author{
Emil Namor, Fabrizio Sossan, Enrica Scolari, Rachid Cherkaoui, Mario \\ Paolone
}

\section{To cite this version:}

Emil Namor, Fabrizio Sossan, Enrica Scolari, Rachid Cherkaoui, Mario Paolone. Experimental Assessment of the Prediction Performance of Dynamic Equivalent Circuit Models of Grid-connected Battery Energy Storage Systems. 2018 IEEE PES Innovative Smart Grid Technologies Conference Europe (ISGT-Europe), Oct 2018, Sarajevo, Bosnia and Herzegovina. pp.1-6. hal-02108794

\author{
HAL Id: hal-02108794 \\ https://hal.science/hal-02108794
}

Submitted on 24 Apr 2019

HAL is a multi-disciplinary open access archive for the deposit and dissemination of scientific research documents, whether they are published or not. The documents may come from teaching and research institutions in France or abroad, or from public or private research centers.
L'archive ouverte pluridisciplinaire HAL, est destinée au dépôt et à la diffusion de documents scientifiques de niveau recherche, publiés ou non, émanant des établissements d'enseignement et de recherche français ou étrangers, des laboratoires publics ou privés. 


\title{
Experimental Assessment of the Prediction Performance of Dynamic Equivalent Circuit Models of Grid-connected Battery Energy Storage Systems
}

\author{
Emil Namor, Fabrizio Sossan, Enrica Scolari, Rachid Cherkaoui, Mario Paolone \\ Distributed Electrical Systems Laboratory (DESL) \\ EPFL, École Polytechnique Fédérale de Lausanne, Switzerland \\ \{emil.namor, fabrizio.sossan, enrica.scolari, rachid.cherkaoui, mario.paolone\}@epfl.ch
}

\begin{abstract}
The paper discusses the model identification, validation and experimental testing of current-to-voltage dynamic circuit models for a grid-connected MW-class battery. The model refers to an utility-scale $720 \mathrm{kVA} / 560 \mathrm{kWh}$ battery energy storage system (BESS) and is used in a model predictive control framework to forecast the evolution of the battery DC voltage as a function of the current trajectory. The model is identified using measurements from a dedicated experimental session where the BESS is controlled with a pseudo random binary signal (PRBS) to excite the system on a broad spectrum. The identified model relies on the assumption that the battery is a single cell. To test this assumption and assess the quality of predictions, we test the model performance by using a second data set coming from a real-life power system application, where the BESS is used to dispatch the operation of a group of stochastic prosumers (demand and PV generation). Experimental results show that the root mean square voltage prediction error of the best performing model (i.e. two time constant model, TTC) is less than $0.55 \%$ for look-ahead times in the range 10 seconds-10 minutes and better than persistence for all considered forecasting horizons.
\end{abstract}

\section{INTRODUCTION AND CONTRIBUTIONS}

Predictive control coupled with forecasting of local consumption and generation is considered with increasing interest to tackle the challenges related to power systems operations with high proportion of production from renewables. In the context of battery energy storage systems (BESSs), model predictive control has been proposed to smooth, dispatch and schedule generation and demand, see e.g. [1]-[4], while efficiently handling BESS's constraints, like managing the battery state of charge (SOC) and respecting current and voltage constraints on the DC bus.

In the existing technical literature, a considerable research effort is devoted to identifying models of electrochemical storage, with the main objective of improving the physical representativeness of model parameters (i.e., an enabling factor to model ageing processes), e.g., [5]-[9]. Another class of models is the family of so-called equivalent circuit models, see e.g. [10]-[12], which consists of an electric circuit with multiple RC branches in series. In spite of some limitations

This research received funding from the Swiss Competence Center on Energy Research (SCCER-FURIES). given in the following section, they are widely adopted in practical applications because they are able to capture dynamics quite accurately and, compared to more detailed models, are tractable.

In this paper, we assess the prediction performance of current-to-voltage equivalent circuit models in a practical grid application. First, we identify equivalent circuit models of an utility-scale BESS from experimental measurements. The models are identified by applying grey-box modeling, a methodology to identify and validate dynamic models of a system incorporating its physical knowledge together with measurements from a real device [13]. Second, we perform an extensive validation of the models' prediction performance in a real-life grid application, where the BESS is controlled by applying model predictive control (MPC) to provide active power dispatch to the grid.

The contribution of this work is illustrating a state-of-the art procedure to identify equivalent circuit models of batteries and documenting their prediction performance in a practical power system application.

This paper is organized as follows: Section II describes the experimental framework, Section III the adopted greybox modelling strategy and identified models, Section IV the results of the identification process, and Section $\mathrm{V}$ the validation results. Finally, Section VI states the conclusions.

\section{EXPERIMENTAL FRAMEWORK AND APPLICATION}

The BESS for which dynamic prediction models are identified is a $560 \mathrm{kWh}$ unit connected to the MV grid of the EPFL campus through a $720 \mathrm{kVA}$ four quadrant power converter. The battery's cells are installed in a temperature controlled container. The main characteristics of the system are summarized in Table I. The grid-connected BESS implements active/reactive power set points, which are communicated over Modbus with a non-deterministic refresh rate of approximately $500 \mathrm{~ms}$. Through the same communication interface, measurements of the voltage and current on the BESS's DC bus and estimations of the SOC are available.

The BESS is the controllable element of the "dispatchable feeder" application, originally described in [3]. In a nutshell, it 
TABLE I

SPECIFICATIONS OF THE EXPERIMENTAL BATTERY ENERGY STORAGE SYSTEM

\begin{tabular}{|c|c|c|}
\hline Parameter & Unit & Value \\
\hline Nominal Power & $\mathrm{kVA}$ & 720 \\
Energy Capacity Power & $\mathrm{kWh}$ & 560 \\
Voltage at the AC connection point & $\mathrm{kV}$ & 20 \\
Number of racks & - & 9 in parallel \\
Number of modules per rack & - & 15 in series \\
Cells configuration per module & - & $20 \mathrm{~s} 3 \mathrm{p}$ \\
Total number of cells & - & 8100 \\
Cell minimum voltage & $\mathrm{V}$ & 1.7 \\
Cell nominal voltage & $\mathrm{V}$ & 2.3 \\
Cell maximum voltage & $\mathrm{V}$ & 2.7 \\
Cell nominal capacity & $\mathrm{Ah}$ & 30 \\
\hline
\end{tabular}

consists in dispatching the operation of a distribution network node (with non-controllable distributed renewable generation and non-shiftable demand) according to a pre-established trajectory, called dispatch plan, i.e. a sequence of average power flow value at 5 minute resolution determined the day before operation according to prosumption forecasts and battery SOC.

As described in the following, the proposed models are identified from measurements taken from a dedicated experimental session, where the BESS is operated in order to excite a wide range of its dynamics. Therefore, models predictions are tested by using the experimental data coming from the dispatchable feeder application with the specific objective of assessing model prediction performance in a real-life application.

\section{GREY-BOX MODELING APPLIED TO EQUIVALENT} CIRCUIT MODEL IDENTIFICATION OF BATTERY SYSTEMS

Dynamic model identification is carried out by applying grey-box modeling on voltage and current measurements on the battery DC bus. Grey-box modeling is a set of rigorous and systematic tools to identify and validate the model structure and parameters from measurements. For our specific case, it consists in the steps described in the following paragraphs.

a) Experiment design and measurement acquisition: A series of dedicated off-line experiments are performed, where the BESS real power flow is controlled with a pseudo random binary signal (PRBS), a two levels square wave with on-off periods of random durations capable of exciting a wide range of system dynamics. Since model parameters are affected by the C-rate, choosing an identification signal of similar amplitude as the one used in the final application is the key to achieve reliable voltage predictions. Also, model parameters strongly depend on the BESS SOC. Therefore, we perform a number of PRBS sessions where the BESS is kept in a specific SOC interval $(0-20 \%, 20-40 \%, 40-60 \%, 60-80 \%, 80-100 \%)$, and, for each interval, a model is estimated. Measurements of the current and voltage at the BESS DC terminals are acquired through the battery Modbus interface at 1 second resolution. They are shown in Fig. 1. Ideally, measurements should be divided into a training and validation data set, the former used to fit model parameters and perform preliminary validation tests, and the latter for advanced validation. In this case, the validation data set is composed of measurement coming from the dispatchable feeder application, which allows validating model prediction performance in practice, i.e. in a real power system application.

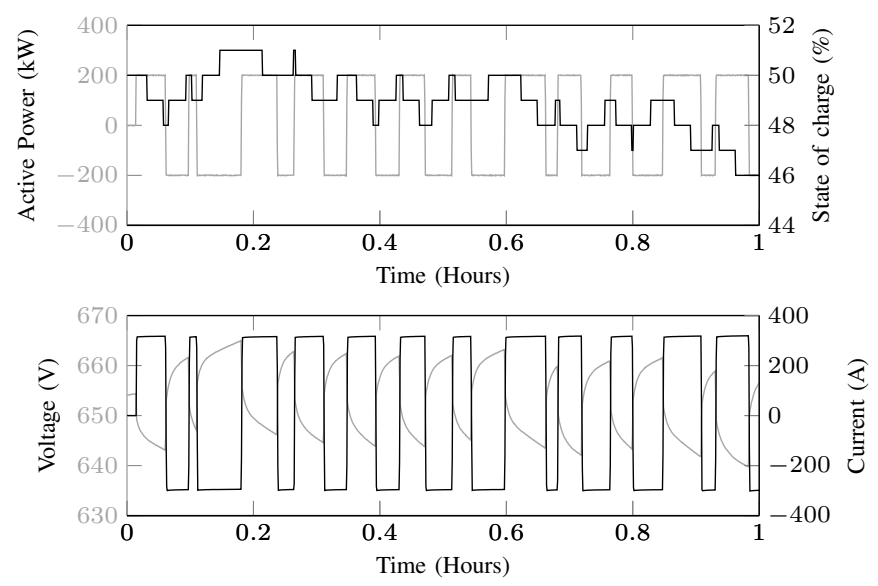

Fig. 1. Identification data set: PRBS active power reference (upper panel), and DC voltage and current measurements (bottom panel).

b) Model Formulation: A set of mathematical relationships is formulated to describe the physical process to model. This constitutes the so-called candidate model. Battery voltage models for control applications normally consist in electric equivalent circuits, which trade detailed modelling of the electrochemical reactions for increased tractability, see for example [10], [11], [14]. The model structure we investigate in this paper is inspired from the well-known two-time constant (TTC) model. It consists in two series RC branches, where the values of model parameters depend on the battery SOC, cells temperature, and C-rate. In the proposed model, the dependency between parameters and SOC is captured by performing the parameter estimation for different SOC's ranges. 1colorblack In the proposed experimental setup, the battery is installed in a temperature controlled environment. By neglecting self-heating, we assume that the temperature is uniform for all the cells and we do not model its impact on the value of models' parameters. The dependency of models' parameters on C-rate is neglected because the BESS is operated with a similar C-rate as used in the real operation. Models are formulated by adopting the stochastic continuoustime state-space representation:

$$
\begin{aligned}
& d x=\mathcal{A}_{c}(\theta) x d t+\mathcal{B}_{c}(\theta) u(t) d t+\mathcal{K}_{c}(\theta) d \omega \\
& v_{k}=\mathcal{C} x_{k}+\mathcal{D}(\theta) u_{k}+\mathcal{G}(\theta) g_{k},
\end{aligned}
$$

where $v_{k}$ is the model output and BESS terminal voltage, $x \in \mathbb{R}^{n}$ system state vector, $n$ model order, $\mathcal{A}_{c}$ system matrix, $\mathcal{B}_{c}$ input matrix, $\mathcal{K}_{c}$ input disturbance matrix, $C$ output matrix, $D$ feedforward matrix, $\mathcal{G}$ measurement noise matrix, $g_{k}$ i.i.d. (independent and identically distributed) standard 
normal noise, $u$ input vector, $\omega$ a $n$-dimension standard Wiener process, and $\theta$ is the set of model parameters to estimate. The terms associated to process and measurements noise vectors $\mathcal{K}_{c}$ and $\mathcal{G}$ are necessary to implement Kalman filtering for state reconstruction. This is required in practical applications because the state vector $x$ is a modelling abstraction, thus not measurable.

c) Parameters estimation: Parameters of the candidate model are estimated by applying maximum likelihood estimation (MLE) on the model one-step-ahead prediction error, implemented in the Matlab function greyest.

d) Model validation: Two validation tests are considered in this modelling effort. First, we verify whether the candidate model can capture all the time dynamics contained in the training data set by evaluating residuals correlation in the model's one-step-ahead prediction errors. These ideally should not contain any predictable structure and be an i.i.d. process. Second, for those parameters with a physical value, we compare the output of the numerical estimation against the empirical physical estimation.

e) Model extension: If the prediction errors are correlated in time, an alternative model should be formulated by, e.g., increasing the order of the candidate model or adopting an alternative mathematical description of the process. The extended candidate model should be estimated and validated by reiterating the procedures described at the points $c$ ) and $d$ ). The model extension can be cross-validated against the older model by applying, for example, the deviance test, to avoid model overfitting due to the increased number of parameters. Nevertheless, this last process is not performed here because the autocorrelation analysis was conclusive already.

\section{Modelling Results and Discussion}

When using 1 second sampled measurements to fit the model, the traditional TTC model was found not capable of absorbing all the dynamics contained in the training data set. The best performing model is the third order linear circuit shown in Fig. 2. It is a TTC model augmented with an additional series $\mathrm{RC}$ branch and a controlled voltage source $E$, described in details later in this section.

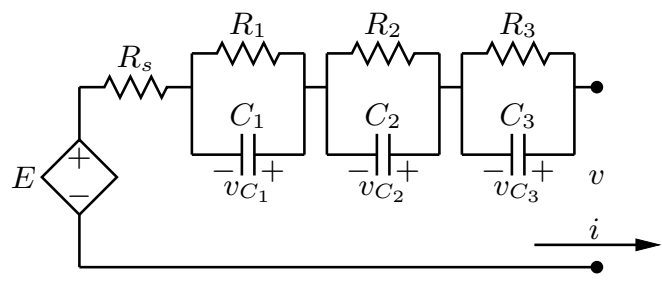

Fig. 2. Best performing model when considering identification measurements at 1 second resolution: $v$ and $i$ are the BESS terminal voltage and DC current, respectively, while $v_{C_{1}}, v_{C_{2}}, v_{C_{3}}$ are the state vector components $x$. 1colorblack $R_{x}, C_{x}$ and open circuit voltage $E$ are parameters the values of which are to identify from measurements as explained in the main text.

The autocorrelation function (ACF) of the third order model's prediction errors for the case of 40-60\% SOC's range and 1 second measurements is shown in Fig. 3, where the unitary value at zero time lag is omitted to better visualize the remaining autocorrelation structure. As visible from Fig. 3, all the ACF samples fall inside the $95 \%$ confidence level of white noise's autocorrelation (dashed lines), thus indicating that the model is able to absorb short-term time dynamics. An equivalent behaviour was observed for the other considered SOC's ranges.

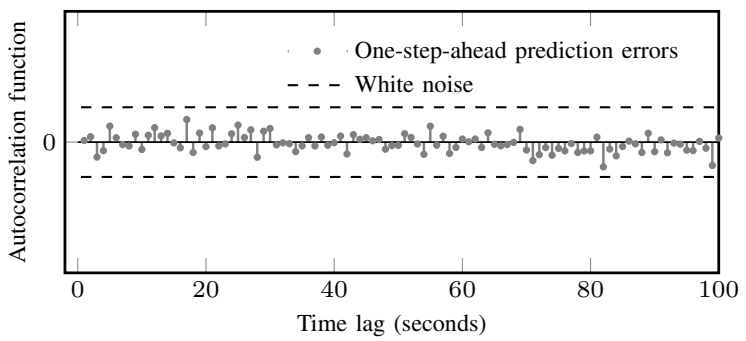

Fig. 3. Autocorrelation function (ACF) of model residuals (full line) and white noise (dashed lines) at $95 \%$ confidence level. The component with unitary value at zero time lag has been removed for a better visual rendering of the remaining autocorrelation structure.

However, since in the practical control application, equivalent circuit models might be applied at a lower pace, the model identification is performed a second time considering measurements at 10 seconds resolution. At this time interval, two time constants were enough to capture all the dynamics contained in the training data set. In other words, the additional state of the third order model was found unnecessary because it referred to dynamics which were not any longer observable in the measurements at lower resolution. The best performing model is shown in Fig. 4, where the controlled voltage source $E$ represents the open circuit voltage of the battery, which, as known, depends on the battery SOC. Even if, in the current modelling effort, the SOC was discretized into ranges (i.e. $0-20 \%, 20-40 \%, 40-60 \%, 60-80 \%, 80-100 \%$ ) to capture the model parameters' dependency on the battery SOC, the variations of the open circuit voltage inside each range is still present and modelling it as a constant value would result in large errors. Therefore, we model it as a linear function of the battery SOC:

$$
E(\operatorname{SOC}(t))=\alpha+\beta \cdot \operatorname{SOC}(t),
$$

where $\alpha$ and $\beta$ are parameters to identify. The deterministic skeleton of the state-space model is derived by applying Kirchhoff laws to the circuit in Fig. 2. To include (3) in the final state-space model, it is convenient to express the SOC with the integral state:

$$
\operatorname{SOC}(t)=\frac{1}{3600 Q} i(t)
$$

where $Q$ is the total battery nominal capacity in Ampere-perhour, and form an augmented state space model, as shown in the following. With reference to the stochastic state-space description introduced in (1)-(2), let the model state and model 
input be:

$$
\begin{aligned}
& x(t)=\left[\begin{array}{lll}
v_{C_{1}}(t) & v_{C_{2}}(t) & \operatorname{SOC}(t)
\end{array}\right]^{T} \\
& u(t)=\left[\begin{array}{ll}
i(t) & 1
\end{array}\right]^{T} .
\end{aligned}
$$

The augmented model matrices are:

$$
\begin{aligned}
& \mathcal{A}_{c}=-\operatorname{diag}\left(\frac{1}{R_{1} C_{1}}, \frac{1}{R_{2} C_{2}}, 0\right) \\
& \mathcal{B}_{c}=\left[\begin{array}{cc}
\frac{1}{C_{1}} & 0 \\
\frac{1}{C_{2}} & 0 \\
\frac{1}{Q} & 0
\end{array}\right] \\
& \mathcal{K}_{c}=\operatorname{diag}\left(k_{1}, k_{2}, k_{3}\right) \\
& \mathcal{C}=\left[\begin{array}{lll}
1 & 1 & \beta
\end{array}\right] \\
& \mathcal{D}=\left[\begin{array}{ll}
R_{s} & \alpha
\end{array}\right] \\
& \mathcal{G}=\sigma_{g} \text {. }
\end{aligned}
$$

where $R_{1}, C_{1}, R_{2}, C_{2}, R_{s}, \alpha, \beta$ (i.e., values of the circuit components), $k_{1}, k_{2}, k_{3}$ (i.e. components of the system noise matrix), and $\sigma_{g}$ (i.e., standard deviation of the measurement Gaussian noise) are the parameters to be estimated. The estimated values of the model parameters are reported in the first six columns of Table II as a function of the BESS SOC range. As a further validation test, the last column of Table II reports the estimated values of the model parameters for the $80-100 \%$ SOC range when the value of $R_{s}$ is assigned instead of being free. The series resistance $R_{s}$ is empirically estimated by evaluating the ratio

$$
R_{s}=\left|\frac{\Delta V}{\Delta I}\right|
$$

where $\Delta V$ is the instantaneous voltage variation occurring in correspondence to a large step in the input current and $\Delta I$ is the magnitude of such current step. As visible from the last two columns of Table II, the parameters' values before and after assigning $R_{s}$ are similar, denoting that the estimation results are stable.

Table III compares, for each considered SOC range, the $R_{s}$ 's values obtained in the MLE-based model identification and empirical estimation (13). Identified and empirical estimated values are similar, thus denoting that the proposed identification process is robust.

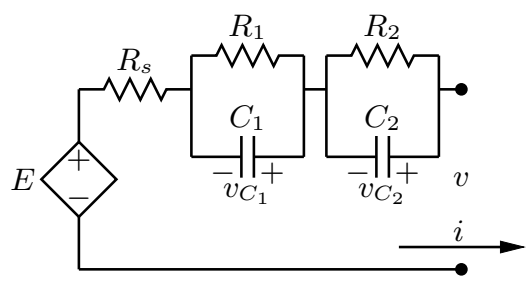

Fig. 4. Best performing model when considering identification measurements at 10 second resolution.

\section{Testing and Validation Results}

In this section, we test the prediction performance of the considered models by using measurements from an existing experimental setup, where the same BESS used for the identification experiment is utilized in a real life application to dispatch the operation of a distribution feeder, as introduced in Section II.

\section{A. Prediction performance}

We test the prediction performance of the identified models on different forecasting horizons (i.e. 10, 30, 60, 120, 180, 300,600 seconds) and compare it against a persistent predictor, i.e. where the point predictions for the next time step is the latest available observation. The performance metric is the percentage root mean square of the voltage prediction error:

$$
\text { percentage } \operatorname{RMSE}(\%)=\sqrt{\frac{1}{N} \sum_{i=1}^{N}\left(\frac{V_{i}-\widehat{V}_{i}}{V_{i}}\right)^{2}} \cdot 100
$$

where $V_{i}$ is the voltage measurements at time step $i=$ $1, \ldots, N$, and $\widehat{V}_{i}$ the voltage prediction.

Eq. (14) is computed for the proposed model and persistent model considering a validation data set of 20 thousands current/voltage measurements at $10 \mathrm{~s}$ resolution, corresponding to 2 contiguous days of battery operation. At each time step, the correct set of parameters is chosen according to the measured SOC. The model (7)-(12) is discretized and used to calculate the voltage prediction as a function of the battery current value. Kalman filtering is applied to estimate and propagate the value of the state as a function of the observed battery voltage, known from measurements.

The predictions of the proposed model and persistent model are shown in Fig. 5. They refer to 10 minutes-ahead prediction horizon and qualitatively show the better accuracy of the former model.
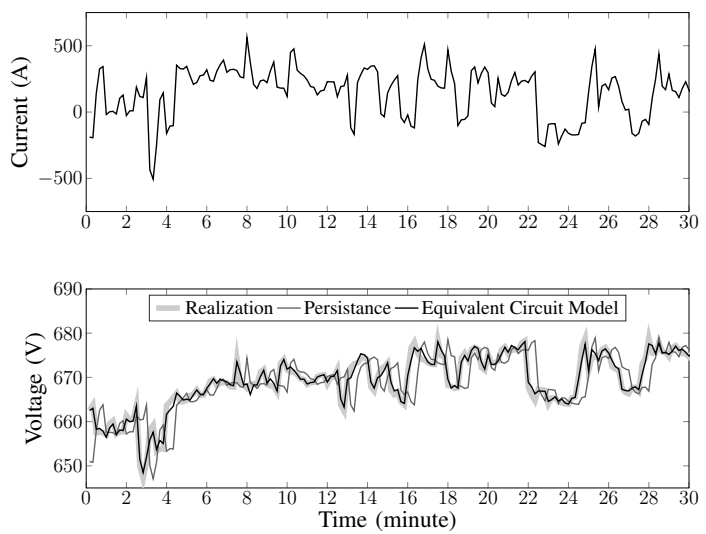

Fig. 5. Thirty seconds-ahead voltage predictions of equivalent circuit and persistent models compared to the realization.

The comparison between the prediction performance of the persistent model and proposed model is shown in Fig. 6, in black and gray, respectively. It can be noted that the percentage RMSE of the proposed model is small (less than $0.55 \%$ ) and 
TABLE II

ESTIMATED BESS VOLTAGE MODEL PARAMETERS FOR DIFFERENT SOC RANGES

\begin{tabular}{|c|c|c|c|c|c|c|}
\hline SOC & $\mathbf{0 - 2 0 \%}$ & $\mathbf{2 0 - 4 0 \%}$ & $\mathbf{4 0 - 6 0 \%}$ & $\mathbf{6 0 - 8 0 \%}$ & $\mathbf{8 0 - 1 0 0 \%}$ & $\mathbf{8 0 - 1 0 0 \%}\left(R_{s}=0.016\right)$ \\
\hline$\alpha$ & 590 & 607.2 & 607.1 & 590 & 590 & 590 \\
$\beta$ & 364.8 & 181.5 & 203.2 & 258.4 & 257.4 & 264.9 \\
$R_{s}$ & 0.083 & 0.023 & 0.018 & 0.017 & 0.015 & $\mathbf{0 . 0 1 6}$ \\
$R_{1}$ & 0.262 & $8.76 \cdot 10^{-3}$ & $7.29 \cdot 10^{-3}$ & $8.78 \cdot 10^{-3}$ & $8.65 \cdot 10^{-3}$ & $8.82 \cdot 10^{-3}$ \\
$C_{1}$ & 1383 & 2254 & 3177 & 3001 & 2682 & 2684 \\
$R_{2}$ & $3.63 \cdot 10^{-5}$ & $2.55 \cdot 10^{-4}$ & $2.28 \cdot 10^{-5}$ & $3.06 \cdot 10^{-4}$ & $4.61 \cdot 10^{-5}$ & $2.42 \cdot 10^{-5}$ \\
$C_{2}$ & $1 \cdot 10^{6}$ & $1 \cdot 10^{6}$ & $1 \cdot 10^{6}$ & $1 \cdot 10^{6}$ & $1 \cdot 10^{6}$ & $1 \cdot 10^{6}$ \\
$k_{1}$ & -9.118 & 0.035 & 0.107 & 0.103 & -0.115 & -1.86 \\
$k_{2}$ & 9.199 & 15.85 & 0.037 & -0.042 & 0.167 & 1.937 \\
$k_{3}$ & $2.5 \cdot 10^{-5}$ & -0.162 & $-4.95 \cdot 10^{-3}$ & $3.09 \cdot 10^{-5}$ & $1.2 \cdot 10^{-4}$ & $3.61 \cdot 10^{-5}$ \\
$\sigma_{g}^{2}$ & 1.998 & 0.215 & 0.035 & 0.025 & 0.035 & 0.05 \\
\hline
\end{tabular}

TABLE III

VALUES OF $R_{s}$ : MAXIMUM LIKELIHOOD ESTIMATION (ML) VERSUS EMPIRICAL ESTIMATION (ES)

\begin{tabular}{|c|ccccc|}
\hline SOC range & $0-20 \%$ & $20-40 \%$ & $40-60 \%$ & $60-80 \%$ & $80-100 \%$ \\
\hline MLE & 0.083 & 0.022 & 0.018 & 0.017 & 0.015 \\
ES & 0.077 & 0.022 & 0.017 & 0.016 & 0.016 \\
\hline
\end{tabular}

its predictions outperform those of the persistent model for all the considered prediction horizons.

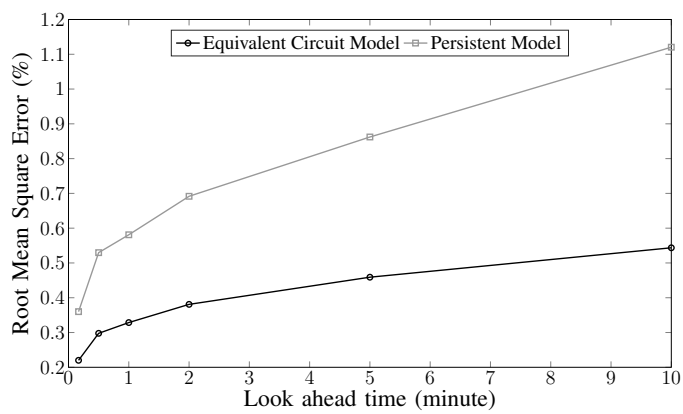

Fig. 6. Percentage root mean square of the prediction errors of the equivalent proposed model and persistent model for look-ahead-times from 10 seconds to 10 minutes.

\section{Vi. Conclusions}

We discussed grey-box modeling applied to the identification of dynamic DC current-to-voltage equivalent circuit models of grid-connected battery systems. Models are estimated from measurements using the information available from a grid-connected $720 \mathrm{kVA} / 560 \mathrm{kWh}$ battery. Models are formulated using the stochastic state-space representation. Their structure is inspired by the well-established TTC model, a linear second order model that originally consists in a voltage source, a series resistance and two parallel RC branches in series. In linear models, Models' parameters are known to depend on SOC, C-rate and temperature values. To capture the first dependency, we have identified several models, each corresponding to a different SOC range (i.e., 0-20, 20-40, 40$60,60-80,80-100 \%)$. For the second, parameters were identified for a value of $\mathrm{C}$-rate similar to the one used in the real application. 1colorblack Finally, the third was not modelled explicitly because the experimental unit under consideration is installed in a temperature controlled environment (i.e., a common design choice for utility-scale battery systems) and self-heating of cells was neglected.

Identification results show that when using measurements sampled at 1 second resolution, a third order model was necessary to capture early dynamics, whereas at 10 second, a second-order model was enough to capture all the dynamics contained in the measurements. In all the models, it has been found useful to augment the state-space with an integral state to represent the battery SOC and express the open circuit voltage as its linear function.

Models' prediction performance was tested and validated on different look-ahead times (i.e., ranging from 10 seconds to 10 minutes) using data from a real power system applications where the battery system is used to dispatch a distribution system with stochastic injections. It was observed that the relative root mean square prediction error of the best performing models is less than $0.55 \%$ for all look-ahead times and less than persistent predictions.

1 colorblack In spite of the simplicity of equivalent circuit models, validation results in an experimental setting with an utility-scale battery energy storage system showed that they can deliver accurate predictions of the voltage on the DC bus as a function of the demanded current. Due to their tractability (inear), ease of identification, and ability of capturing voltage dynamics, their use is convenient in control applications. A limitation of the current modelling framework is that parameters' values are piecewise constant according to the SOC's range, thus generating discontinuity in the estimation. The hypothesis to curve-fit parameters' values was in general not viable as they lacked a well identifiable trend.

\section{REFERENCES}

[1] C. T. Li, H. Peng, and J. Sun, "Mpc for reducing energy storage requirement of wind power systems," in 2013 American Control Conference, 
June 2013, pp. 6607-6612.

[2] M. Abu Abdullah, K. Muttaqi, D. Sutanto, and A. Agalgaonkar, "An effective power dispatch control strategy to improve generation schedulability and supply reliability of a wind farm using a battery energy storage system," Sustainable Energy, IEEE Transactions on, vol. 6, 2015.

[3] F. Sossan, E. Namor, R. Cherkaoui, and M. Paolone, "Achieving the dispatchability of distribution feeders through prosumers data driven forecasting and model predictive control of electrochemical storage," IEEE Transactions on Sustainable Energy, vol. 7, no. 4, Oct 2016.

[4] E. Namor, F. Sossan, R. Cherkaoui, and M. Paolone, "Load leveling and dispatchability of a medium voltage active feeder through battery energy storage systems: Formulation of the control problem and experimental validation," in 2016 IEEE PES Innovative Smart Grid Technologies Conference Europe (ISGT-Europe), Oct 2016.

[5] K. A. Smith, C. D. Rahn, and C.-Y. Wang, "Control oriented 1d electrochemical model of lithium ion battery," Energy Conversion and Management, vol. 48, no. 9, pp. 2565-2578, 2007.

[6] D. Di Domenico, G. Fiengo, and A. Stefanopoulou, "Lithium-ion battery state of charge estimation with a kalman filter based on a electrochemical model," in 2008 IEEE International Conference on Control Applications. Ieee, 2008, pp. 702-707.

[7] A. Bizeray, S. Zhao, S. Duncan, and D. Howey, "Lithium-ion battery thermal-electrochemical model-based state estimation using orthogonal collocation and a modified extended kalman filter," Journal of Power Sources, vol. 296, pp. 400-412, 2015.

[8] R. D. Perkins, A. V. Randall, X. Zhang, and G. L. Plett, "Controls oriented reduced order modeling of lithium deposition on overcharge," Journal of Power Sources, vol. 209, pp. 318-325, 2012.

[9] A. V. Randall, R. D. Perkins, X. Zhang, and G. L. Plett, "Controls oriented reduced order modeling of solid-electrolyte interphase layer growth," Journal of Power Sources, vol. 209, pp. 282-288, 2012.

[10] M. Chen and G. Rincon-Mora, "Accurate electrical battery model capable of predicting runtime and i-v performance," Energy Conversion, IEEE Transactions on, vol. 21, June 2006.

[11] B. Y. Liaw, G. Nagasubramanian, R. G. Jungst, and D. H. Doughty, "Modeling of lithium ion cells-a simple equivalent-circuit model approach," Solid State Ionics, vol. 175, 2004.

[12] C. Birkl and D. Howey, "Model identification and parameter estimation for lifepo 4 batteries," in Hybrid and Electric Vehicles Conference 2013 (HEVC 2013), IET. IET, 2013, pp. 1-6.

[13] F. Sossan, V. Lakshmanan, G. T. Costanzo, M. Marinelli, P. J. Douglass, and H. Bindner, "Grey-box modelling of a household refrigeration unit using time series data in application to demand side management," Sustainable Energy, Grids and Networks, vol. 5, pp. 1-12, 2016.

[14] M. Bahramipanah, D. Torregrossa, R. Cherkaoui, and M. Paolone, "Enhanced electrical model of lithium-based batteries accounting the charge redistribution effect," in 2014 Power Systems Computation Conference, Aug 2014, pp. 1-8. 